\title{
The barriers of clinical education in nursing: A systematic review.
}

\author{
Hosien Shadadi $^{1}$, Mahmood Sheyback ${ }^{2}$, Abbas Balouchi ${ }^{1,3}$, Maryam Shoorvazi ${ }^{*}$ \\ ${ }^{1}$ Department of Nursing, School of Nursing and Midwifery, Zabol University of Medical Sciences, Zabol, Iran \\ ${ }^{2}$ Student Research Committee, Bushehr University of Medical Sciences, Bushehr, Iran \\ ${ }^{3}$ Nursing Care Research Center, School of Nursing and Midwifery, Iran University of Medical Sciences, Tehran, Iran \\ ${ }^{4}$ Department of Nursing, School of Nursing, Neshabur University of Medical Sciences, Neshabur, Iran
}

\begin{abstract}
Considering the great significance of proper clinical education in training nurses qualifying for provision of quality care, this study has been conducted with the aim of investigating the obstacles against clinical education among the nurses. In this systematic review study, international databases (PubMed, Web of Science, Scopus, CINHAL, EMBASE, ERIC, Google scholar, as well as national banks including Magiran and SID) were searched from the time of inception until January 30, 2018. The obstacles of clinical education in individual areas (associated with students (Lack of motivation in students), professors (Absence of experience professor with a high academic level), and nurses (Personnel uncooperativeness)), managerial (Shortage of time), facilities (Deficit of facilities and working conditions), structures, and other areas were identified. Based on the obstacles identified across various dimensions, proper plans and strategies should be designed and implemented with each of the obstacles to enhance the quality of clinical education.
\end{abstract}

Keywords: Clinical education, Nursing education, Barriers, Systematic review.

Accepted on October 16, 2018

\section{Introduction}

Today, considering the change in learning needs, changes of the pattern of diseases, and increased care needs, on the one hand, and the shortage of nurses as the largest part of the healthcare team as well as very high cost in nursing and medical education, on the other, the importance of caring for proper clinical education has been multiplied [1-6]. Proper clinical education alongside theoretical education is one of the most important parts of education in nursing, in which the care behaviors of nurses are formed [6-10]. Also, the objective of nursing which is providing proper high-quality care is realized through this $[1,11-13]$. Clinical practice is considered an opportunity for learning skills by nurses, which unlike theoretical classes, it is a very complex context packed with challenges in different dimensions [14-16]. Proper educational setting in clinical practice is one of the very important factors in the learning of students and nurses to provide quality care to patients [17-20]. Therefore, understanding the obstacles against clinical education can result in better and faster recognition of the obstacles against clinical education, finding solutions, and resolving them through enhancing learning quality, preventing expenditure of nonessential costs, and determining better strategies based on the recognized obstacles [21-29]. The studies conducted in this area suggest that most studies have investigated the obstacles individually, and only in a few of them which have reviewed the literature of obstacles of clinical learning, the most important obstacles included time and financial constraints as well as lack of access to evidencebased literature [18,30-33]. Considering the crucial importance of understanding the factors and search by the researcher, so far no study has been conducted as systematic review to examine the obstacles against clinical education as the most important dimension of nursing education to train qualified nurses for providing quality healthcare services [34-36]. This systematic review study has been conducted with the aim of investigating the obstacles against clinical education of nurses.

\section{Method}

\section{Inclusion criteria}

This study has been composed based on the guideline for conducting systematic review studies (Cochran) and using PRISMA checklist [37]. This study protocol registered in PROSPERO (ID: CRD42018096394). Inclusion criteria were included: all observational studies (descriptive, descriptive analytical, case-control, and cohort) published in peerreviewed journals were included. Only the papers in Persian and English were included. All the studies which had examined the obstacles of clinical education from the view of nurses, professors, and students of nursing with quantitative 
approaches were included. The minimum sample size of studies included should have been at least 25 . The main objective in this study was to examine the obstacles against clinical education, while the secondary objective was to offer suggestions to reduce clinical education obstacles.

\section{Search strategy}

In this study, international databases (PubMed, Web of Science, Scopus, Cumulative Index to Nursing and Allied Health Literature (CINHAL), The Excerpta Medica database (EMBASE), Education Resources Information Center (ERIC), Google scholar) as well as national databases (Magiran, Scientific Information Database (SID)) were searched from the time of inception until January 30, 2018. The utilized keywords were determined based on Medical Subject Headings (MESH) and Emtree, and then merged together using NOT, AND, and OR operators. The utilized keywords in Persian and English included obstacles, barriers, clinical education, clinical learning, and nursing.

\section{Selecting the studies and extracting information}

According to the study protocol and the inclusion criteria, two researchers investigated the title and abstract of the studies separately, and then the repeated cases were eliminated. In the next stage, the full text of the papers was examined and the necessary information was extracted. In cases where disagreement occurred for selecting the studies, consensus method was used to resolve the disagreement between the two writers. The items of the extracted information included general information (first author, year, country, sample size, data collection method) and implication (the utilized instrument, obstacles of clinical education, and suggestions).

\section{Quality of studies}

Investigation of the quality of studies was performed using strengthening the Reporting of Observational Studies in Epidemiology (STROBE) standard instrument [38] which is used for observational studies. Guided by the 22 items a score was assigned independently to each study. Scores were reviewed, and discrepancies were resolved by consensus. Studies with scores of 14 or below out of 22 possible points were categorized as low quality; those with scores of $15-17$ as medium quality, and studies with scores of 18 or higher were categorized as high quality.

\section{Results}

\section{General results}

Selection of studies: Overall, based on the primary search across different databases, 1748 papers were found, out of which 1534 were non-repeated. In the next stage, the nonrepeated papers were investigated based on their title and abstract, and 1504 papers were removed due to their incongruence with the objective of this study and inclusion criteria. In the next stage, 30 papers were investigated as full- text, where 10 studies entered the final stage. Twenty papers were excluded at this stage, where 7 were review, 11 were qualitative, and one lacked full text. One paper had a target population other than healthcare team (Figure 1).

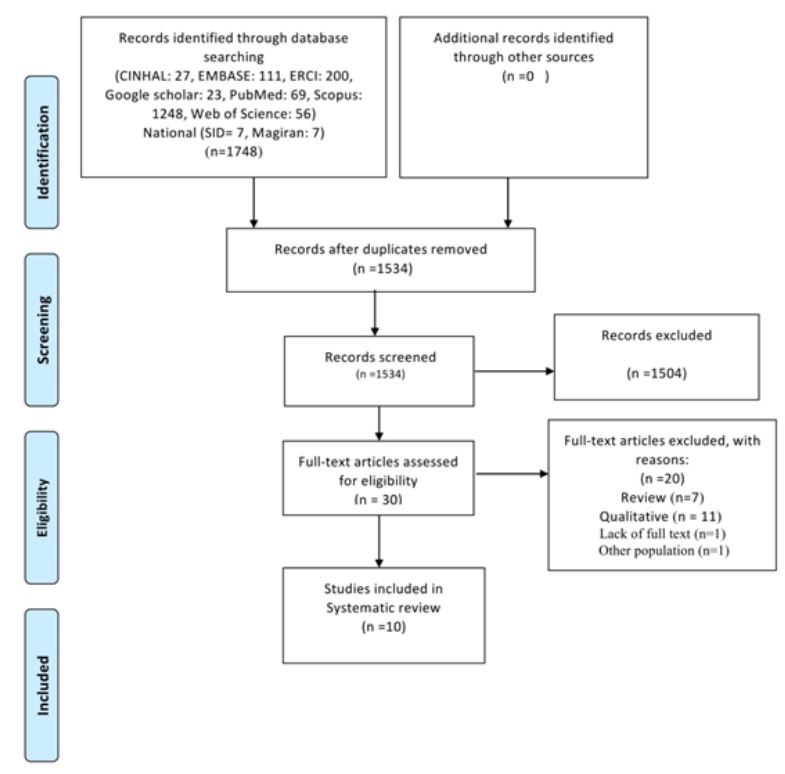

Figure 1. Study selection process.

The characteristics of the included studies: All of the included studies had been conducted in Iran in their final stage $(n=10)$ and descriptively on 1468 participants. The mean age of the participants was 26.59 (8.1 y old). The sampling had been conducted as census $(n=8)$ in most of the studies (Table 1). All of the included studies had a quality above $50 \%$.

Instruments: Most of the instruments used in the present study have been researcher made, which had been developed based on investigating the literature and by the researchers. Only two studies had used standard instruments $[13,39]$. The number of items in the instruments was 20-59. Further, in two studies, the number of items had not been mentioned properly [40,41]. The validity of the instruments had been investigated by $9-10$ experts. Further, regarding the reliability of the instruments, the Cronbach alpha ranged from 0.75 to 0.94 across different instruments [13,40-48] (Table 2).

\section{The obstacles of nursing clinical education}

Defining the obstacles of clinical education in nursing is very important and lead to increase of quality of education in clinical nursing cares. The obstacles mentioned in different studies varied considerably. However, based on main factors, they can be divided into four areas: individual (the obstacles associated with student, professor, and nurses), management, facilities, and others. In the area of individual factors, the most important obstacle against clinical education related to the students based on the different studies was lack of motivation in the students $[40,41]$. The most important obstacles against clinical education associated with professors included shortage of experienced professors with a high academic level, not stating the educational objectives for the students, and not 
assessing the students' activities by trainers based on internship objectives $[13,40,41,45,47]$. The most important obstacles against clinical education associated with nurses included inadequate knowledge and skill and uncooperativeness of the personnel $[13,40,41,43,48]$. In the area of management, the most important obstacles against clinical education included the mismatch between clinical education objectives and the expectations of the hospital personnel and shortage of time $[13,43,44,47]$. In the area of facilities and structures, the most important obstacles against clinical education included shortage of facilities and their working conditions, lack of access to conference room, and poor educational planning [13,39-41,43-45,47,48]. Other clinical education obstacles included not recognizing the role of nurses as teachers for patients and the society, as well as uncooperativeness of the patient and neglecting the education (Table 3) [42].

The frequent solutions presented for reducing the obstacles against clinical education included: developing proper facilities and equipment in clinical centers, increasing the motivation of students, and taking measures to incorporate clinical education in nursing curricula (Table 2).

Table 1. The general characteristics of the studies included in the systematic review.

\begin{tabular}{|c|c|c|c|c|c|c|}
\hline First author (year) & Country & Study design & Study participants & Age & Gender (male/female) & Sampling method \\
\hline Salehabadi [41] & Iran & Descriptive & 129 & 22.19 & $23 / 93$ & Census \\
\hline Dehghani [42] & Iran & $\begin{array}{l}\text { Descriptive } \\
\text { sectional }\end{array}$ & 271 & 29.6 & $53 / 218$ & Census \\
\hline Gholami [43] & Iran & Descriptive & 95 & 25.15 & $38 / 57$ & Census \\
\hline Heidari [44] & Iran & $\begin{array}{l}\text { Descriptive } \\
\text { sectional }\end{array}$ & 150 & 21.58 & $51 / 99$ & Census \\
\hline Jahanpour [45] & Iran & $\begin{array}{l}\text { Descriptive } \\
\text { sectional }\end{array}$ & 58 & Not mentioned & $35 / 21$ & Census \\
\hline Jahromi [13] & Iran & $\begin{array}{l}\text { Descriptive } \\
\text { sectional }\end{array}$ & 78 & 21.66 & $47 / 31$ & Census \\
\hline Moghimi [47] & Iran & $\begin{array}{l}\text { Descriptive } \\
\text { sectional }\end{array}$ & 108 & 22.37 & $86 / 22$ & Census \\
\hline Rahimi [40] & Iran & $\begin{array}{l}\text { Descriptive } \\
\text { sectional }\end{array}$ & 38 & 40.5 & 29-Sep & Random \\
\hline Rezaei Nik [48] & Iran & Descriptive - analytical & 384 & $20-40$ & $274 / 66$ & Multistage randomized \\
\hline \multirow[t]{2}{*}{ Tanomand [39] } & Iran & Descriptive - analytical & 157 & $\begin{array}{l}34.64 \\
\text { professors }\end{array}$ & $17 / 33$ & Census \\
\hline & & & & 23.18 students & $18 / 89$ & \\
\hline
\end{tabular}

Table 2. The utilized instruments, obstacles, and suggestions for resolving the obstacles against clinical education in nursing.

\begin{tabular}{|c|c|c|}
\hline \multirow[t]{4}{*}{ Author } & The utilized instrument & \multirow[t]{4}{*}{ Suggestions } \\
\hline & 1. Type and components & \\
\hline & 2. Number of items & \\
\hline & 3. Reliability and validity & \\
\hline \multirow[t]{3}{*}{ Salehabadi [41] } & $\begin{array}{l}\text { 1. Researcher made questionnaire, including three } \\
\text { sections: demographic information, problems of } \\
\text { clinical education, and solution for improving it for } \\
\text { trainers and students. }\end{array}$ & Creating a balance in the number of students in internship groups \\
\hline & 2. Has not been mentioned. & Making the students aware about the consequences resulting from mistakes \\
\hline & $\begin{array}{l}\text { 3. The instrument was provided for } 20 \text { students } \\
\text { and } 10 \text { faculty members, and the reliability was } \\
\text { confirmed by a Cronbach alpha of } 0.85 \text {. }\end{array}$ & Assessing the students for relegating tasks \\
\hline \multirow[t]{2}{*}{ Dehghani [42] } & $\begin{array}{l}\text { 1. Researcher made consisting of two sections: } \\
\text { demographic characteristics and the obstacles of } \\
\text { education to the patient. }\end{array}$ & \\
\hline & 2. 33 items & Providing a sufficient number of personnel, pri \\
\hline
\end{tabular}


3. It was given to 10 faculty members and the developing the culture for approving education by patients reliability was confirmed by Cronbach alpha of 0.91 .

\begin{tabular}{|c|c|c|}
\hline \multirow[t]{3}{*}{ Gholami [43] } & $\begin{array}{l}\text { 1. Researcher made consisting of two parts } \\
\text { (demographic-assessing the opinions of students } \\
\text { about the obstacles against clinical education) }\end{array}$ & $\begin{array}{l}\text { Most of these obstacles can be amended and by resolving these obstacles, one can } \\
\text { achieve improved performance of students and eventually provision of effective and safe } \\
\text { care. }\end{array}$ \\
\hline & 2. 59 & \\
\hline & $\begin{array}{l}\text { 3. It was given to } 10 \text { faculty members and the } \\
\text { reliability was confirmed by Cronbach alpha of } 0.94 \\
\text { and } 0.84 \text {. }\end{array}$ & \\
\hline \multirow[t]{3}{*}{ Heidari [44] } & 1. Researcher made & $\begin{array}{l}\text { Since the opinions of students can effectively contribute to enhancing the quality of } \\
\text { clinical education, the nursing students' opinions should be measured periodically }\end{array}$ \\
\hline & 2. 31 & \\
\hline & $\begin{array}{l}\text { 3. It was given to } 10 \text { faculty members and the } \\
\text { reliability was confirmed with a Cronbach alpha of } \\
0.93 \text {. }\end{array}$ & \\
\hline \multirow[t]{2}{*}{ Jahanpour [45] } & $\begin{array}{l}\text { 1. Researcher made-demographic and obstacles } \\
\text { of clinical education in pediatric ward }\end{array}$ & \\
\hline & 2. 30 & $\begin{array}{l}\text { It is suggested that the relevant authorities and planners of nursing education take steps } \\
\text { to improve the motivation and learning of students using the research results by } \\
\text { identifying the obstacles ahead of clinical education of the pediatric ward for students } \\
\text { and trainers. }\end{array}$ \\
\hline
\end{tabular}

3. It was given to 10 faculty members, and the reliability was confirmed by Cronbach alpha 0.91 .

$\begin{array}{ll}\text { Jahromi [13] } & \begin{array}{l}\text { 1. Standard-including the obstacles and facilitators } \\ \text { of clinical education }\end{array} \\ & \begin{array}{l}\text { The educational methods are recommended to be used three useful strategies. The } \\ \text { seriously in academic curricula. }\end{array}\end{array}$

\section{20 items}

3. for obstacles: 0.75 ; for facilitators: 0.78 .

Moghimi [47] 1. Researcher made-demographic, factors, and obstacles

2. 20 items

Reviewing and taking suitable measures by academic authorities seem to be essential for a suitable clinical education setting including facilities and equipment of clinical setting and reviewing the instruments and processes of clinical education

3. It was given to 10 experts and the reliability was confirmed with a Cronbach alpha of 0.81 .

Rahimi [40] 1. Researcher made including four sections: More attention by nursing planners and authorities for proper planning for clinical demographic, clinical obstacles, solutions, and practice problems).

2. Not mentioned exactly

Employing experienced and skilful trainers

3. It was given to nine nursing experts, and the Encouraging and developing interest in students reliability was confirmed by Cronbach alpha of 0.89

One of the most important current obstacles is individual obstacles. To reduce or improve this challenge, cooperation should be developed between trainer, clinical nurses, academic supervisor, head nurses, nursing managers, the educational planner, and other top-level management authorities.

\section{26}

3. It was given to 10 nursing experts, where the Cronbach alpha reliability for obstacles and facilitators was 0.78 and 0.86 , respectively.

Tanomand [39] 1. Standard

The facilities and equipment of clinical setting and reviewing the system of recording and reporting according to nursing standards should be improved. 
3. The reliability was confirmed by Cronbach alpha of 0.87 .

Table 3. Classifying the obstacles against clinical education.

\begin{tabular}{|c|c|c|}
\hline Dimensions of obstacles & Sources & Obstacles \\
\hline \multirow[t]{23}{*}{ Individual dimension } & Student & Lack of motivation in students $[40,41]$ \\
\hline & & Lack of proper communication between students and the ward personnel [47] \\
\hline & & Not adhering to order and discipline by the student [47] \\
\hline & & Lack of proper communication between students and professor [47] \\
\hline & Professor & Absence of experience professor with a high academic level $[13,40,41,47]$ \\
\hline & & Lack of suitable motivation in trainers [41] \\
\hline & & Emphasizing theoretical aspects in educational work [41] \\
\hline & & Not stating the educational objectives for students $[45,47]$ \\
\hline & & Being unfamiliar with educational methods [47] \\
\hline & & Absence at essential hours in the ward [47] \\
\hline & & Not assessing the students activities based on the internship objectives by the trainers $[45,47]$ \\
\hline & & Inadequate feedback to students [43] \\
\hline & & Relegating heavy and difficult tasks to students [43] \\
\hline & & Lack of proper communication between students and professor [47] \\
\hline & & Not emphasizing pre-study by trainers [43] \\
\hline & Nurses & Mismatch between the job of personnel and scientific principles [41] \\
\hline & & Not implementing the process by the personnel [41] \\
\hline & & Lack of physical and psychological preparation [13] \\
\hline & & Fatigue [48] \\
\hline & & Inadequate knowledge and skill $[13,40]$ \\
\hline & & Not planning education to the patient in the daily task of nurses as a duty [42] \\
\hline & & Personnel uncooperativeness $[41,43,48]$ \\
\hline & & Improper treatment of the personnel [43] \\
\hline \multirow{9}{*}{\multicolumn{2}{|c|}{ Management dimension }} & Large number of students in internship groups [41] \\
\hline & & Discrimination between nursing students and the students of other medical sciences [41] \\
\hline & & The patient or their companions complaining about performing nursing affairs by students [48] \\
\hline & & Unsuitable internship time [47] \\
\hline & & Mismatch between the objectives of clinical education and expectations of the hospital personnel $[43,44]$ \\
\hline & & Shortage of time $[13,47]$ \\
\hline & & Not prioritizing education in the description of duties [42] \\
\hline & & Not gaining score for the nurse to train the patient [42] \\
\hline & & Mismatch between the number of patients and number of nurses [42] \\
\hline \multirow{2}{*}{\multicolumn{2}{|c|}{ The dimension of facilities and structures }} & Deficit of facilities and working conditions $[13,41,43,45]$ \\
\hline & & Limited cases in the wards [41] \\
\hline
\end{tabular}




\begin{tabular}{ll} 
& The hospital being non-academic [41] \\
\cline { 2 - 2 } Lack of access to the conference room [39,43] \\
\hline Others & Poor educational planning $[40,44,47,48]$ \\
\hline Not recognizing the role of nurses as teachers for patients and the society [10] \\
\hline
\end{tabular}

\section{Discussion}

The present systematic review study was conducted with the aim of investigating the obstacles against clinical education of nursing in internal and foreign databases until January 30, 2018. In the present study, the most important obstacles against clinical education mentioned in different studies were categorized into four groups: individual area (obstacles associated with students, professors, and nurses), management, facilities, and others. Similar studies conducted in the area of obstacles against clinical education were in the form of review of literature. For this reason, after investigating them, the results of qualitative studies have also been used in the discussion. An integrated review study conducted by Santos indicated that the most important obstacles against nurses' learning were time constraints, financial constraints, the culture of the workplace, access, and matching of new technologies to acquire knowledge. In the dimensions of time constraints and culture of workplace, access, and matching through new technologies to acquire knowledge based on evidence, it confirms the results of the present study [1]. Another study conducted by Foster et al about the challenges of clinical education of nurses indicated that the obstacles of clinical education included unclear description of duties which take much of the nurse's time, and instead of dealing with more essential duties, they seek to resolve the peripheral problems of patients; high working load as well as time and financial constraints which prevent use of robust clinical evidence in education, which is in line with the present study [49]. The results of a qualitative study by Abbaszadeh et al. suggested that the most important challenges of clinical education included development of learning potentials in practice (the professor presentation power and student presentation power), confronting the conflicts of real practice (ineffective planning and executive obstacles of acquiring clinical skill), efficient clinical education (assessment-oriented flexible planning, effective clinical professor, and moving beyond dependence towards independence in practice), and professional challenges in the future (professional version and unsafe clinical setting) [2]. In this regard, the results of the present study in individual dimensions related to professor and students confirm the management dimensions. However, it also mentions professional challenges in the future, which were not found in the present study [2]. Another study conducted by Alavi et al. indicated that the most important obstacles against clinical education according to students were tension, conflict, and lack of access to direct experience [50]. Conflict indeed represented problematic communication, which is in line with the results of the present study. However, the dimensions of conflict and lack of direct access to experience were not observed in the present study, which can be due to the qualitative nature of the investigative the study and the different sample size of subjects [50]. The study by Jamshidi et al. showed that the most important obstacles against clinical education were ineffective communication, inadequate preparation for emotional reactions, which regarding ineffective communication and inadequate preparation, it confirms the results of the present study [46]. However, regarding the dimension of emotional reactions which refer to tensions in clinical education, it was not found in the present study [46].

The most important solutions of the present study to reduce the obstacles of clinical education were: creating suitable facilities and equipment in clinical centers, increasing the motivation of the students, and taking measures to incorporate clinical education in nursing curricula, which are in line with the results of different studies $[1,2,46,50]$.

\section{Limitations}

The most important limitations of the present study were:

1. In spite of searching different databases, the information resources in this regard with systematic review approach were limited.

2. In spite of using systematic search strategy with the relevant words, all of the included studies were related to one country.

3. In some cases, the information of the studies was not available. To resolve this, the authors were contacted.

\section{Strengths}

1. The present study is the first systematic review study for this purpose.

2. Use of systematic review approach for searching and organizing studies.

\section{Conclusion}

The present systematic review study indicated that the most important obstacles of learning were categorized individual, management, facilities, and equipment dimensions. Considering the importance of each dimension, regarding the individual dimension associated with students, some plans should be developed to increase the motivation of the students and hold effective communication workshops. More important is employing more experienced professors with a higher academic level in the clinical practice, so that they can 
generate interest in the students effectively to acquire clinical skills. Further, in the dimension of nurses, since they are the so-called best individuals for education because of being in the clinical setting and alongside the patient bed, and it is recommended to care for management dimensions of obstacles and through incorporating the clinical education curriculum into the description of nurses' duties, proper planning of nurses' shifts, and granting score to individuals interested in clinical education, their attention to clinical education should be improved. In the management dimension, the most important strategy can be proper planning for the number of nursing students in the right wards, so that all the students can equally use the opportunities developed in the clinical practice for learning. In the dimension of equipment and facilities, the necessary financial resources for equipping the libraries of hospitals and creating evidence-based centers through new technologies should be taken seriously.

\section{References}

1. Santos MC. Nurses barriers to learning: an integrative review. J Nurses Staff Dev 2012; 28: 182-185.

2. Abbaszade A, Borhani F, Sabzevari S. Nursing teachers' perception of the challenges of clinical education and solutions: a qualitative study. Qual Res Health Sci 2013; 2: $134-145$.

3. Lapkin S, Levett-Jones T. A cost-utility analysis of medium vs. high-fidelity human patient simulation manikins in nursing education. J Clin Nurs 2011; 20: 3543-3452.

4. Jones-Schenk J, Leafman J, Wallace L, Allen P. Addressing the cost, value, and student debt in nursing education. Nurs Econ 2017; 35: 7-13, 29.

5. Jeffries PR, Jeffries PR. Simulation in nursing education: From conceptualization to evaluation: National League for Nursing New York, NY 2012.

6. Benner P. Educating nurses: a call for radical transformation-how far have we come? J Nurs Educ 2012; 51: 183-184.

7. Butcher HK, Bulechek GM, Dochterman JMM, Wagner C. Nursing Interventions classification (NIC)-E-Book: Elsevier Health Sciences 2018.

8. Edvardsson D, Watt E, Pearce F. Patient experiences of caring and person-centredness are associated with perceived nursing care quality. J Adv Nurs 2017; 73: 217-227.

9. Gantt LT, Webb-Corbett R. Using simulation to teach patient safety behaviors in undergraduate nursing education. J Nurs Educ 2010; 49: 48-51.

10. Pardue KT, Morgan P. Millennials considered: a new generation, new approaches, and implications for nursing education. Nurs Educ Perspect 2008; 29: 74-79.

11. Suikkala A, Koskinen S, Leino-Kilpi H. Patients involvement in nursing students clinical education: A scoping review. Int J Nurs Stud 2018; 84: 40-51.
12. Altmiller G, Armstrong G. 2017 National Quality and Safety Education for Nurses Faculty Survey Results. Nurse Educ 2017; 42: S3-3S7.

13. Jahromi ZB. A study of the barriers and facilitators of patient education from the viewpoint of nursing students at Jahrom college of nursing. Bangladesh J Med Sci 2016; 15: 471-476.

14. Higgs J, Jones MA, Loftus S, Christensen N. Clinical reasoning in the health professions E-Book: Elsevier Health Sciences 2018.

15. Clark CM, Farnsworth J, Landrum RE. Development and description of the incivility in nursing education (INE) survey. J Theor Construct Test 2009; 13: 7.

16. Bell-Scriber MJ. Nursing education research: warming the nursing education climate for traditional-age learners who are male. Nurs Educ Perspect 2008; 29: 143-150.

17. Jefferies D, McNally S, Roberts K, Wallace A, Stunden A, DSouza S. The importance of academic literacy for undergraduate nursing students and its relationship to future professional clinical practice: a systematic review. Nurse Educ Today 2017; 60: 84-91.

18. Thomas LJ, Asselin M. Promoting resilience among nursing students in clinical education. Nurse Educ Pract 2018; 28: 231-234.

19. Papp I, Markkanen M, von Bonsdorff M. Clinical environment as a learning environment: student nurses perceptions concerning clinical learning experiences. Nurs Educ Today 2003; 23: 262-268.

20. Tanner CA. The next transformation: clinical education. J Nurs Educ 2006; 45: 99-100.

21. Perkins MB, Jensen PS, Jaccard J, Gollwitzer P, Oettingen G, Pappadopulos E. Applying theory-driven approaches to understanding and modifying clinicians behavior: what do we know? Psych Serv 2007; 58: 342-348.

22. Grol R, Grimshaw J. From best evidence to best practice: effective implementation of change in patients care. Lancet 2003; 362: 1225-1230.

23. Lambert V, Glacken M. Clinical education facilitators: a literature review. J Clin Nurs 2005; 14: 664-673.

24. Mangena A, Chabeli MM. Strategies to overcome obstacles in the facilitation of critical thinking in nursing education. Nurse Educ Today 2005; 25: 291-298.

25. Mikkonen K, Elo S, Kuivila HM, Tuomikoski AM, Kaariainen M. Culturally and linguistically diverse healthcare students experiences of learning in a clinical environment: a systematic review of qualitative studies. Int J Nurs Studies 2016; 54: 173-187.

26. Chen MJ, Yu S, Chen IJ, Wang KWK, Lan YH, Tang FI. Evaluation of nurses knowledge and understanding of obstacles encountered when administering resuscitation medications. Nurse Educ Today 2014; 34: 177-184.

27. Vezeau TM. In defense of clinical conferences in clinical nursing education. Nurs Educ Pract 2016; 16: 269-273.

28. Reyes AT, Andrusyszyn MA, Iwasiw C, Forchuk C, Babenko-Mould Y. Nursing students understanding and 
enactment of resilience: a grounded theory study. J Adv Nurs 2015; 71: 2622-2633.

29. Cunningham J, Wright C, Baird M. Managing clinical education through understanding key principles. Radiol Technol 2015; 86: 257-273.

30. Mackey A, Bassendowski S. The history of evidencebased practice in nursing education and practice. J Prof Nurs 2017; 33: 51-55.

31. Goudreau J, Pepin J, Larue C, Dubois S, Descôteaux R, Lavoie P. A competency-based approach to nurses continuing education for clinical reasoning and leadership through reflective practice in a care situation. Nurse Educ Pract 2015; 15: 572-578.

32. Forsgren S, Christensen T, Hedemalm A. Evaluation of the case method in nursing education. Nurse Educ Pract 2014; 14: 164-169.

33. Foronda CL, Baptiste DL, Pfaff T, Velez R, Reinholdt M, Sanchez M. Cultural competency and cultural humility in simulation-based education: an integrative review. Clin Simul Nurs 2018; 15: 42-60.

34. Forbes H, Oprescu FI, Downer T, Phillips NM, McTier L, Lord B, Barr N, Alla K, Bright P, Dayton J, Simbag V, Visser I. Use of videos to support teaching and learning of clinical skills in nursing education: a review. Nurse Educ Today 2016; 42: 53-56.

35. Ironside PM, McNelis AM, Ebright P. Clinical education in nursing: rethinking learning in practice settings. Nurs Outlook 2014; 62: 185-191.

36. Papastavrou E, Dimitriadou M, Tsangari H, Andreou C. Nursing students satisfaction of the clinical learning environment: a research study. BMC Nurs 2016; 15: 44.

37. Moher D, Shamseer L, Clarke M, Ghersi D, Liberati A, Petticrew M. Preferred reporting items for systematic review and meta-analysis protocols (PRISMA-P) 2015 statement. Sys Rev 2015; 4: 1 .

38. Von Elm E, Altman DG, Egger M, Pocock SJ, Gøtzsche PC, Vandenbroucke JP. The Strengthening the Reporting of Observational Studies in Epidemiology (STROBE) statement: guidelines for reporting observational studies. PLoS Med 2007; 4: 296.

39. Tanomand A, Niknam F. Evaluation of environmental barriers in clinical education in viewpoints of instructors and nursing students. Educ Develop Jundishapur 2014; 5: 106-113.

40. Rahimi A, Ahmadi F. The obstacles and improving strategies of clinical education from the viewpoints of clinical instructors in Tehrans nursing schools. Iran J Med Educ 2005; 5: 73-80.

41. Salehabadi S, Akbarzadeh M, Nasrollahi S, Golafroz shahri M, Mircholi N. Clinical education problems and ways of enhancing its quality from the perspective of clinical instructors and students of nursing and midwifery at Sabzevar university medical sciences in 2008. J Sabzevar Uni Med Sci 2015; 20: 539-546.

42. Dehghani A, Orang M, Abdollahyfard S, Parviniyan Nasab AM, Vejdani MA. Barriers to patient education in clinical care viewpoints of nurses. Iran J Med Educ 2014; 14: 332-341.

43. Gholami H, Ahmadi chenari H, Chamanzari H, Shakeri M. Amendable barriers in Clinical education from Viewpoints of students and clinical instructors at faculty of nursing and midwifery of Mashhad. J Torbat Heydariyeh Univ Med Sci 2015; 3: 34-39.

44. Heidari MR, Norouzadeh R. Nursing students perspectives on clinical education. $\mathrm{J}$ Adv Med Educ Professional 2015; 3: 39-43.

45. Jahanpour F, Zarei A, Ravanipour M, Razazan N, Hoseini S. Nursing students views on problems in clinical education in the pediatric ward in Bushehr University of Medical Sciences. J Nurs Edu 2014; 2: 72-79.

46. Jamshidi N, Molazem Z, Sharif F, Torabizadeh C, Kalyani $\mathrm{MN}$. The challenges of nursing students in the clinical learning environment: a qualitative study. Sci World J 2016; $2016: 7$.

47. Moghimi M, Mohammad Hossini S, Karimi Z, Moghimi M, Naimi E, Mohammadi S. Obstacles of clinical education and strategies for the improvement of quality of education in Yasuj university of medical sciences. J Res Develop Nurs Midwifery 2014; 11: 95-101.

48. Rezaei Nik B, Rassuli M, Vaskooi Eshkvari K, Alavi Majd H, Torabi F. Evaluating the obstacles and facilitators of clinical nurses cooperation in clinical education of nursing students. J Nurs Edu 2017; 6: 56-64.

49. Foster J, Flanders S. Challenges in clinical nurse specialist education and practice. Online J Issues Nurs 2014; 19: 1.

50. Alavi M, Irajpour A, Nasiri A, Abedi H. Barriers to clinical education: Student nurses Experiences. Mod Care J 2009; 6: 5-11.

\section{*Correspondence to}

Maryam Shoorvazi

Department of Nursing

School of Nursing

Neshabur University of Medical Sciences

Iran 